\title{
DCPA: approximate adder design exploiting dual carry prediction
}

\author{
Woong Choi ${ }^{1 \dagger}$, Minseob Shim ${ }^{2 \dagger}$, Hyelin Seok ${ }^{3}$, and Yongtae Kim ${ }^{3 a)}$
}

\begin{abstract}
This letter presents a novel approximate adder that significantly improves computation accuracy by utilizing a dual carry prediction and error reduction scheme. In our experiments, the proposed adder improves mean error distance (MED) and mean relative error distance (MRED) by up to $58.6 \%$ and $58.5 \%$, respectively, when compared with existing approximate adders. Also, when implemented in 65- $\mathrm{nm}$ CMOS technology, the proposed adder reduces area, delay, and power by $37 \%, 48 \%$, and $41 \%$, respectively, compared with the traditional adder. Furthermore, the effectiveness of our design over existing adders is investigated using a digital image processing application.

key words: approximate adder, dual carry prediction, energy efficiency Classification: Integrated circuits (memory, logic, analog, RF, sensor)
\end{abstract}

\section{Introduction}

Recently, approximate computing, which trades computation accuracy for energy efficiency, has been investigated for use in error-resilient applications, such as image processing and machine learning $[1,2,3,4,5]$. The opportunity for efficiency lies in the fact that these applications do not require full precision accuracy but can tolerate a certain amount of errors in computations. One of the most important and heavily used arithmetic operations is addition and approximate adders improve energy efficiency by reducing hardware complexity in approximate computing $[6,7,8]$. Therefore, an efficient approximate adder is the key enabler and a considerable number of related works have been presented in the literature $[9,10,11,12,13,14,15,16,17,18,19,20,21,22,23,24]$. One of the most efficient approximate adder architecture is to split an adder into two parts: accurate and inaccurate parts $[9,13]$. In general, the former yields accurate outputs for higher-order bits using a precise adder, such as ripple carry adder (RCA), whereas the latter yields approximate outputs for lower-order counterparts by utilizing approximate logics. Additionally, to improve overall computation accuracy, the

\footnotetext{
${ }^{\dagger}$ These authors contributed equally to this work

${ }^{1}$ Department of Electronics Engineering, Sookmyung Women's University, Seoul 04310, Korea

${ }^{2}$ Korea Electrotechnology Research Institute (KERI), Changwon, Gyeongnam 51543, Korea

${ }^{3}$ School of Computer Science and Engineering, Kyungpook National University, Daegu 41566, Korea

a) yongtae@knu.ac.kr
}

DOI: $10.1587 /$ elex.18.20210431

Received October 11, 2021

Accepted October 25, 2021

Publicized November 02, 2021 accurate part often uses a carry input predicted by the inaccurate part [9]. This architecture was adopted by the lowerpart OR adder (LOA), and the OR operation is used to approximately add lower-order bits with the AND-based carry prediction [9]. The optimized lower-part constant OR adder (OLOCA) is identical to the LOA except that a few least significant bit (LSB) outputs are set to 1 regardless of the corresponding inputs, resulting in hardware cost savings with a slight degradation in accuracy [10]. The hardware optimized adder having a near-normal error distribution (HOAANED) is a design variant of the OLOCA that improves error characteristics [11]. The approximate mirror adder 5 (AMA5) utilizes a mirror operation for both approximate addition and carry prediction [12]. The the error tolerant adder I (ETAI) uses a modified XOR operation without a carry prediction, whereas the simplified ETA (SETA) optimized the ETAI's operation to decrease hardware complexity [13, 14].

In this letter, we present an approximate adder that uses a dual carry speculation, which utilizes two input pairs to predict the carry, to significantly improve the accuracy. Also, the proposed error reduction scheme improves error characteristics using only a few logic gates. To demonstrate the efficacy of our design, we systematically examine it and extensively compare it to existing adders in terms of hardware resource, computation accuracy, and practical application.

\section{Proposed approximate adder design}

Fig. 1 shows the proposed adder architecture, termed dual carry prediction adder (DCPA). The $n$-bit adder divides a $k$-bit accurate part to yield higher-order accurate outputs $S_{n-1: n-k}$ and carry output $C_{\text {out }}$ using a precise adder and an $(n-k)$-bit inaccurate part to obtain lower-order approximate ones $S_{n-k-1: 0}$, which is done by the OR operation except for its most significant bit (MSB), where the XOR is used to improve the accuracy by forming a half adder. Also, the constant truncation for $l$ LSBs is employed to reduce hardware complexity. While the conventional LOA and its variants use only $(n-k-1)^{\text {th }}$ input to predict a carry for the accurate part, in our design, its previous $(n-k-2)^{t h}$ input is also utilized to enhance the prediction accuracy. The carry $C_{i n 1}$ generated from $(n-k-1)^{t h}$ input is directly fed into the precise adder. The other carry $C_{n-k-2}$ generated from $(n-k-2)^{t h}$ input should be propagated through $(n-k-1)^{t h}$ bit position to pass it to the accurate part. Since an XOR plays a role of the propagate signal, there- 


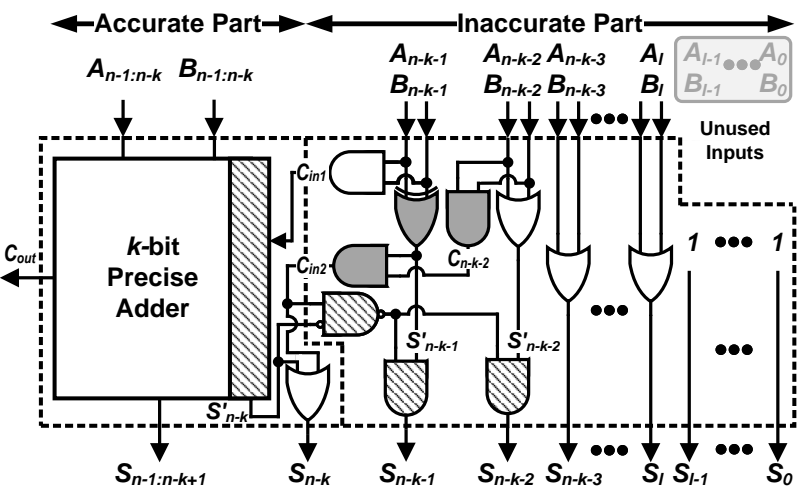

Fig. 1. Proposed approximate adder architecture.

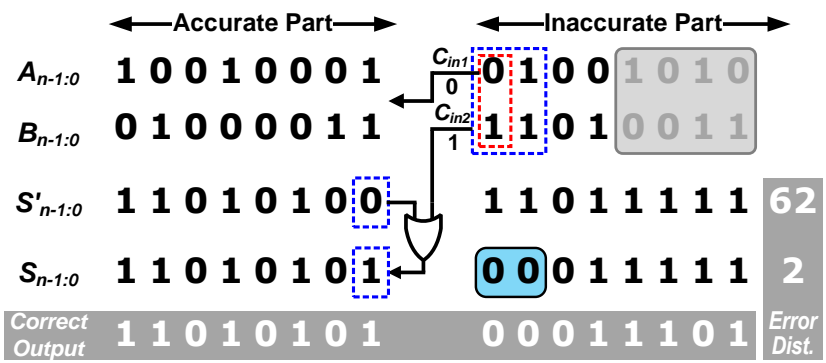

Fig. 2. An example operation of the proposed adder when $C_{i n 2}=1$.

fore, $C_{n-k-2}$ and $(n-k-1)^{t h}$ XOR output are ANDed to produce the second carry $C_{i n 2}$ (see solid-filled gates). Importantly, to avoid an increase in the adder's delay, this carry is ORed with the precise adder's LSB output to produce the final LSB (i.e., $S_{n-k}=C_{i n 2}$ OR $S_{n-k}^{\prime}$ ). Moreover, the error reduction logic (see pattern-filled gates) decreases the error distance (ED) metrics when the second carry $C_{i n 2}$ is 1 .

Fig. 2 illustrates an operation of our 16-bit adder with $k=8$ and $l=4$ when $C_{i n 2}=1$. The accurate part adds $k$ MSB inputs and the carry $C_{i n 1}$ to produce an intermediate output $S_{n-1: n-k}^{\prime}$. The inaccurate part produces a lower-order intermediate output $S_{n-k-1: 0}^{\prime}$ by conducting XOR/OR operations for the higher four bits with constant truncation for the lower four. Then, the second carry $C_{i n 2}$ is ORed with the precise adder's LSB output to produce the final LSB output $S_{n-k}$. When $C_{i n 2}=1$, the intermediate output $S_{n-k-1: n-k-2}^{\prime}$ is always $11_{2}$ but its correct output is $00_{2}$. Therefore, the error reduction logic decreases the ED by forcing the output to $00_{2}$. Note that this reduction occurs when $S_{n-k}^{\prime}=0$ because $C_{i n 2}$ does not affect the accurate part's output when $S_{n-k}^{\prime}=1$. Also, this logic does not affect the output when $C_{i n 2}=0$ and therefore, the intermediate output becomes the final. Under a given input in Fig. 2, the ED and relative ED (RED) are significantly reduced from 62 to 2 and from $1.14 e-3$ to $3.67 e-5$, respectively, by more than $96 \%$.

\section{Experimental results}

The proposed adder, an accurate adder (RCA), and six existing approximate adders (LOA, OLOCA, HOAANED, AMA5, ETAI, and SETA) were designed in Verilog-HDL and synthesized with a 65- $\mathrm{nm}$ CMOS technology using Syn-
IEICE Electronics Express, Vol.VV, No.NN, 1-3
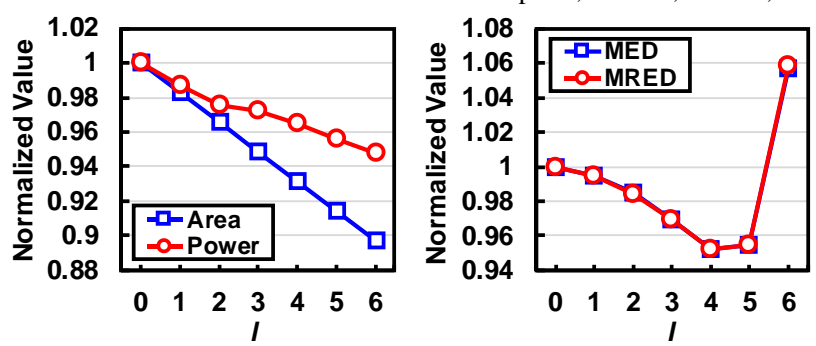

Fig. 3. Performance analysis of the proposed adder according to $l$ values.

Table I. Performance summary of various adders.

\begin{tabular}{c||c|c|c||c|c}
\hline Design & $\begin{array}{c}\text { Area } \\
\left(\mu \mathrm{m}^{2}\right)\end{array}$ & $\begin{array}{c}\text { Delay } \\
(\mathrm{ns})\end{array}$ & $\begin{array}{c}\text { Power } \\
(\mu \mathrm{W})\end{array}$ & MED & $\begin{array}{c}\text { MRED } \\
(1 \mathrm{e}-3)\end{array}$ \\
\hline \hline RCA & 162.6 & 2.27 & 46.2 & - & - \\
\hline LOA & 101.8 & 1.18 & 25.5 & 47.9 & 1.01 \\
\hline OLOCA & $\mathbf{9 0 . 2}$ & 1.18 & $\mathbf{2 4 . 1}$ & 52.0 & 1.09 \\
\hline HOAANED & 93.1 & 1.18 & 24.9 & 32.0 & 0.68 \\
\hline AMA5 & 94.7 & 1.17 & 25.0 & 64.0 & 1.35 \\
\hline ETAI & 113.3 & $\mathbf{1 . 1 2}$ & 27.2 & 51.2 & 1.07 \\
\hline SETA & 97.6 & $\mathbf{1 . 1 2}$ & 24.4 & 55.8 & 1.17 \\
\hline DCPA & 102.1 & 1.18 & 27.3 & $\mathbf{2 6 . 5}$ & $\mathbf{0 . 5 6}$ \\
\hline
\end{tabular}

opsys Design Compiler. We considered 16-bit adders with an 8-bit precise adder (i.e., $n=16$ and $k=8$ ) [10, 25, 26, 27]. Also, the error metrics, such as mean ED (MED) and mean RED (MRED), were extracted under $10^{7}$ random inputs.

Fig. 3 shows the performance analysis of the proposed adder with various $l$ from 0 to 6 . All the values were normalized using the value at $l=0$. The area and power decrease as $l$ increases because a larger $l$ require fewer OR gates. Note that the delay is not affected by $l$ and is consistent. The adder's MED and MRED trend is the same over $l$. They linearly decrease as $l$ increases from 0 to 4 and drastically increase from $l=5$ to $l=6$. The accuracy performance is almost identical at $l=4$ and $l=5$ but a larger $l$ brings a greater hardware benefit. Therefore, $l=5$ can be chosen for the best tradeoff between hardware and accuracy and the proposed adder with $l=5$ will be used for comparison with others.

Table I summarizes the performance of various adders. The RCA has the longest delay due to its long carry chain and consumes the most area and power. Because of its simple approximation with constant truncation, the area and power of the OLOCA are the smallest. The lack of carry prediction makes the ETAI and SETA the fastest adder. The HOAANED and AMA5 use a similar hardware resource, as do the LOA and our DCPA. The MED and MRED values for the OLOCA, ETAI, and SETA are similar, but the LOA's accuracy shows slightly better than these adders. Certainly, our adder design achieves the best in terms of the MED and MRED performance. Specifically, the proposed DCPA outperforms the AMA5 by $2.42 \times$ and $2.41 \times$, respectively.

To assess the efficacy of the proposed adder in practical applications, we applied our design to a $3 \times 3$ edge detection filter in [28]. We filtered the well-known 8-bit grayscale cameraman image using our adder with $n=12, k=6$, and $l=3$ to avoid biased inputs. The same parameters were used for others. The output images filtered using accurate and approximate adders are shown in Fig. 4. The peak signal-to- 


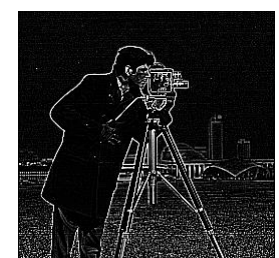

RCA

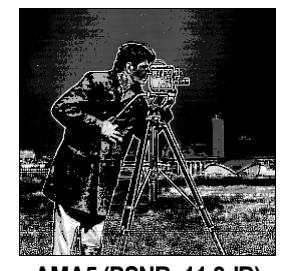

AMA5 (PSNR: 11.8dB)

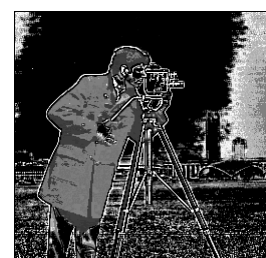

LOA (PSNR: $10.4 \mathrm{~dB}$ )

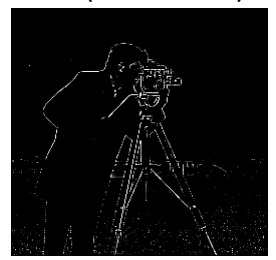

ETAI (PSNR: 15.1dB)

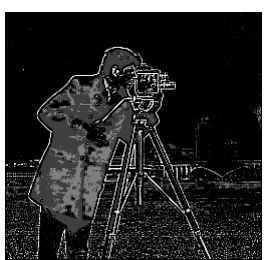

HOAANED (PSNR: 16.7dB)

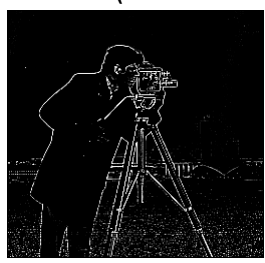

DCPA (PSNR: 19.1dB)
Fig. 4. Output images with PSNRs filtered using various adders.

noise ratio (PSNR) was used to assess the image quality, and the values were extracted against the output image filtered using the accurate adder [29, 30]. The OLOCA and SETA produce nearly identical output images that are visually indistinguishable with a PSNR difference $\leq 0.1 \mathrm{~dB}$ from the LOA and ETAI, respectively, and thus, the images filtered using them were excluded from this analysis. The filtering using the LOA and AMA5 do not properly detect edges and pixel inversions occurred in some regions. The PSNRs of the HOAANED and ETAI are higher, at $16.7 \mathrm{~dB}$ and $15.1 \mathrm{~dB}$, respectively, but there is some noise and unclear edges. The proposed DCPA achieves the best performance with a PSNR of $19.1 \mathrm{~dB}$ in the edge detection because the resultant image is the closest to the one filtered using the accurate adder.

\section{Conclusion}

This letter proposed an approximate adder that utilizes a dual carry prediction to significantly improve the overall accuracy with the error reduction scheme. The proposed adder, when implemented in 65- $\mathrm{nm}$ CMOS technology, reduced area, delay, and power by $37 \%, 48 \%$, and $41 \%$, respectively, and achieved up to a $2.4 \times$ better MED and MRED performance than the AMA5. Furthermore, the efficacy of the proposed design was clearly demonstrated in the edge detection, where our adder outperforms existing approximate adders.

\section{Acknowledgments}

This work was supported by Basic Science Research Program through National Research Foundation of Korea (NRF) funded by the Ministry of Education (NRF2019R1I1A3A01061266) and Institute of Information \& Communications Technology Planning \& Evaluation (IITP) funded by the Korea government (MSIT) (2021-0-00903).

\section{References}

[1] J. Chang, et al:: "An efficient implementation of 2D convolution in CNN," IEICE Electron. Express 14 (2017) 1.

[2] Q. Wang, et al.: "A parallel digital VLSI architecture for integrated support vector machine training and classification," IEEE Trans. Very Large Scale Integr. (VLSI) Syst. 23 (2015) 1471.

[3] Y.-L. Shen, et al.: "Pipelined implementation of AI-based Loeffler DCT," IEICE Electron. Express 10 (2013) 1.
[4] Y.-L. Shen, et al.: "Loeffler DCT accelerator for small portable devices," IEICE Electron. Express 12 (2015) 1.

[5] Y. Kim, et al.: "A reconfigurable digital neuromorphic processor with memristive synaptic crossbar for cognitive computing," ACM J. Emerg. Technol. Comput. Syst. 11 (2015) 38.

[6] D. Liu et al.: "Delay-optimized floating point fused add-subtract unit," IEICE Electron. Express 12 (2015) 1.

[7] B. K. Mohanty: "Efficient fixed-width adder-tree design," IEEE Trans. Circuits Syst. II, Exp. Briefs 66 (2018) 292.

[8] S. Mittal: "A survey of techniques for approximate computing," ACM Comput. Survey 48 (2016) 62.

[9] H. Mahdiani, et al.: "Bio-inspired imprecise computational blocks for efficient VLSI implementation of soft-computing applications," IEEE Trans. Circuits Syst. I, Reg. Papers 57 (2010) 850.

[10] A. Dalloo, et al.: "Systematic design of an approximate adder: the optimized lower part constant-OR adder," IEEE Trans. Very Large Scale Integr. (VLSI) Syst. 26 (2018) 1595.

[11] P. Balasubramanian, et al.: "An approximate adder with a near-normal error distribution: design, error analysis and practical application," IEEE Access 9 (2021) 4518.

[12] V. Gupta, et al.: "Low-lower digital signal processing using approximate adders," IEEE Trans. Comput.-Aided Design Integr. Circuits Syst. 32 (2013) 124.

[13] N. Zhu, et al.: "Design of low-power high-speed truncation-errortolerant adder and its application in digital signal processing," IEEE Trans. Very Large Scale Integr. (VLSI) Syst. 18 (2010) 1225.

[14] J. Lee, et al:: "Approximate adder design with simplified lower-part approximation," IEICE Electron. Express, 17 (2020) 1.

[15] W. Liu, et al.: "Design and analysis of inexact floating-point adders," IEEE Trans. Comput. 65 (2016) 308.

[16] H. Seo, et al.: "Design and analysis of approximate adder with hybrid error reduction," Electronics 9 (2020) 471.

[17] M. Shafique, et al.: "A low latency generic accuracy configurable adder," Proc. Design Autom. Conf. (DAC) (2015) 81.

[18] Y. Kim: "An accuracy enhanced error tolerant adder with carry prediction for approximate computing," IEIE Trans. Smart Process. Comput. 8 (2019) 324.

[19] Y. Kim: "A novel approximate adder with enhanced low-cost carry prediction for error tolerant computing," IEIE Trans. Smart Process. Comput. 8 (2019) 506.

[20] H. Waris, et al.: "High-performance approximate half and full adder cells using NAND logic gate," IEICE Electron. Express 16 (2019) 1.

[21] H. Seo, et al.: "COREA: delay- and energy-efficient approximate adder using effective carry speculation," Electronics 10 (2020) 2234.

[22] Y. Kim, et al.: "Energy efficient approximate arithmetic for error resilient neuromorphic computing," IEEE Trans. Very Large Scale Integr. (VLSI) Syst. 23 (2015) 2733.

[23] T. Sato, et al.: "Trading accuracy for power with a configurable approximate adder," IEICE Trans. Electron. 102 (2019) 260.

[24] T. Yang, et al.: "An accuracy-configurable adder for low-power applications," IEICE Trans. Electron. 103 (2020) 68.

[25] F. Ebrahimi-Azandaryani, et al.: "Block-based carry speculative approximate adder for energy-efficient applications," IEEE Trans. Circuits Syst. II, Exp. Briefs 67 (2019) 137.

[26] J. Lee, et al.: "A novel approximate adder design using error reduced carry prediction and constant truncation," IEEE Access 9 (2021) 1.

[27] B. Garg, et al:: "Reconfigurable carry look-ahead adder trading accuracy for energy efficiency," J. Signal Process. Syst. 93 (2021) 99.

[28] X. Wang: "Laplacian operator-based edge detectors," IEEE Trans. Pattern Anal. Mach. Intell. 29 (2007) 886.

[29] O. Akbari, et al.: "RAP-CLA: a reconfigurable approximate carry look-ahead adder," IEEE Trans. Circuits Syst. I, Reg. Papers 65 (2018) 1089.

[30] H. A. F. Almurib, et al.: "Approximate DCT image compression using inexact computing," IEEE Trans. Comput. 67 (2018) 149. 